\title{
Achromatic dual-zone phase mask stellar coronagraph
}

\author{
R. Soummer ${ }^{1}$, K. Dohlen ${ }^{2}$, and C. Aime ${ }^{1}$ \\ ${ }^{1}$ Laboratoire Universitaire d'Astrophysique de Nice - UMR 6525, Université de Nice Sophia Antipolis, Parc Valrose, \\ 06108 Nice Cedex 2, France \\ e-mail: Claude. Aime@unice.fr \\ ${ }^{2}$ Laboratoire d'Astrophysique de Marseille, 2 place Leverrier, 13248 Marseille, Cedex 4, France \\ e-mail: Kjetil.Dohlen@oamp.fr
}

Received 31 October 2002 / Accepted 14 February 2003

\begin{abstract}
This paper presents a generalization of the Roddier \& Roddier Phase Mask coronagraph for polychromatic observations. It is shown that using a dual-zone phase mask, combined with complex apodization, both phase and size chromatism can be compensated simultaneously to produce high extinction of a point source over large bandwidths, for example the entire $K$ band with a residual integrated starlight of $3.2 \times 10^{-4}$ and a star intensity level of $10^{-6}$ at an angular separation of $3 \lambda / D$. Other advantages of the proposed technique include the compatibility with centrally obscured telescopes, absence of blind axes and no symmetrization of the images.
\end{abstract}

Key words. instrumentation: high angular resolution - techniques: high angular resolution - stars: planetary systems

\section{Introduction}

The scientific objectives of high contrast imaging are numerous, for example exoplanet imaging, circumstellar disks, stellar environnements of massive or evolved stars, Active Galactic Nuclei. For all of these scientific objectives, both imaging and spectral information are essential and polychromatic high contrast images are needed.

Several coronagraphic techniques aim at obtaining these requirements, with different characteristics: the Achromatic Interfero-Coronagraph (AIC) (Gay \& Rabbia 1996; Baudoz et al. 2000a,b) is the only coronagraph totally achromatic by construction but it symmetrizes the images and is therefore not adapted to produce images of complex structures (e.g. protoplanetary disks).

Focal plane mask coronagraphs are more suited for imaging and several techniques have been proposed. The Roddier \& Roddier Phase Mask Coronagraph (Roddier \& Roddier 1997) can yield a theoretical $100 \%$ monochromatic perfect case in its prolate apodized version (PARRC) (Aime et al. 2002; Soummer et al. 2003) but suffers from two chromatism effects: size and phase chromatisms. However it can be used with central obscuration or even diluted apertures (Guyon \& Roddier 2002).

The Prolate Apodized Lyot Coronagraph PALC (Aime et al. 2002; Soummer et al. 2003) is only affected by the size chromatism but needs larger mask sizes, of the order of $3 \lambda / D$ to $4 \lambda / D$ and has a lower throughput (of the order of $20 \%$

Send offprint requests to: $\mathrm{R}$. Soummer,

e-mail: Remi.Soummer@unice.fr to $30 \%$ ). It has been shown that its polychromatic residual intensity remains satisfactory, even without size chromatism correction (Soummer et al. 2002). Its sensitivity to central obscuration is important.

Sectorised phase mask coronagraphs, such as the Four Quadrants Phase Mask FQPM (Rouan et al. 2000; Riaud et al. 2001) or the Phase Knife Coronagraph PKC (Abe et al. 2001, 2003), can also yield a total extinction in the monochromatic perfect case (Abe et al. 2003). These techniques are only affected by the phase shift chromatism. However, they are highly sensitive to pupil shape and pupil remodelling is necessary to achieve optimal performance. In particular, the performance of the quadrant phase mask on a telescope having a central obscuration can be recovered by using a pupil mask that is concave everywhere (Llyod et al. 2002). Also, images suffer from reduced sensitivity along the sector edges (roughly by a factor of 10) which causes loss of observation efficiency, especially for imaging objects very close to the star. This may require rotation of the instrument. Several possibilities for the achromatization of these techniques have been proposed, involving multilayer compensation techniques (Riaud et al. 2001), birefringence (Mawet et al. 2002) or dispersion (Abe et al. 2001).

Unfortunately, none of these techniques presents simultaneously all the advantages: large bandpass coronagraphic efficiency, good imaging capabilities, possible use with central obscuration.

The purpose of this paper is to present an original approach that we consider as a polychromatic generalization of the Roddier \& Roddier Coronagraph. This concept permits one to obtain high contrast over very large bandwidths (up to $40 \%$ 


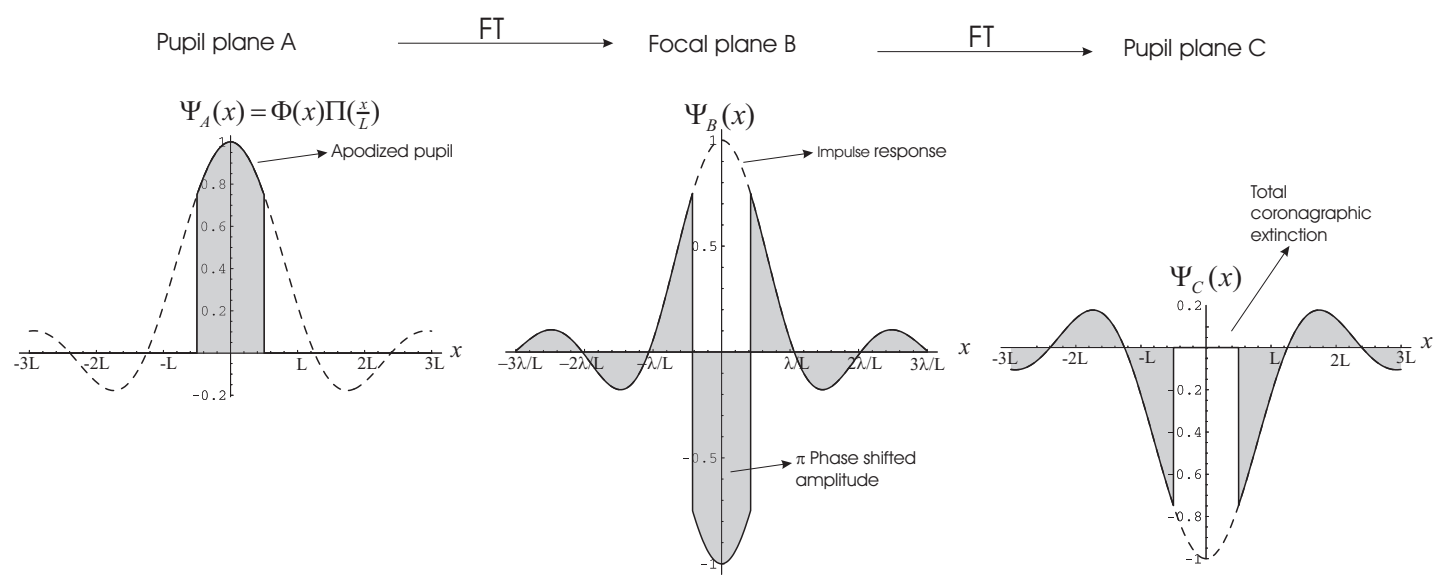

Fig. 1. This figure reviews the principle of the prolate apodized Roddier \& Roddier coronagraph in the monochromatic perfect case. The plots represent the wave amplitudes $\Psi_{\mathrm{A}}(x), \Psi_{\mathrm{B}}(x), \Psi_{\mathrm{C}}(x)$ (dimensionless) as a function of the spatial position $x$ in the three planes A, B, C for a one-dimensional telescope of width $L$. The entrance pupil $\Pi(x / L)$ is apodized by the adequate prolate function $\Phi(x)$ (left figure). The $\pi$ phase mask (multiplication by -1) is applied to the wave amplitude in the focal plane (center figure). The direct wave and the wave diffracted by the mask interfere destructively inside the pupil plane C (right figure). In this perfect case, the Lyot Stop (not represented) eliminates all the star light and the extinction is total. The amplitude in the focal plane D is not represented since it is null in the whole field (total extinction of the star). For more details, refer to Aime et al. (2002) and Soummer et al. (2003).

for example). Moreover, it can be used with a centrally obscured telescope and can provide snapshot images.

The proposed approach consists of re-visiting the chromatism problems associated with phase masks, taking them into account from the beginning. This approach leads to the use of a dual-zone non- $\pi$ phase mask combined with a complexapodized entrance pupil. The possibility of three zones is discussed in the conclusion.

The results hereafter indicate that this method can provide high achromatic nulling over a large band with near-perfect nulling at two wavelengths. In Sect. 2, we introduce the theoretical basis of this method from the analysis of the Roddier $\&$ Roddier Phase Mask chromatism. In Sect. 3 we give the general formalism and results for a circular pupil telescope.

\section{Phase masks coronagraphs and chromatism}

\subsection{Roddier \& Roddier phase mask}

Roddier \& Roddier (1997) have proposed an improvement of Lyot's stellar coronagraph, by replacing the occulting mask by a transparent $\pi$ phase shifting mask, slightly smaller than the Airy disk. This technique can be greatly improved by a slight apodization of the entrance pupil, as proposed by Guyon \& Roddier (2000) and Baudoz (1999). The formal study of this technique (Aime et al. 2002; Soummer et al. 2003) showed that it can yield a theoretical $100 \%$ nulling in the monochromatic case for a perfect telescope, using prolate spheroidal apodizations.

The principle of the Prolate Apodized Roddier \& Roddier Coronagraph is recalled in Fig. 1, adapted from Aime et al. (2002), and involves four planes A, B, C, D. A denotes the telescope aperture (with apodization mask), B denotes the telescope focus (with the coronagraphic phase mask), $\mathrm{C}$ denotes the Lyot pupil plane (with the Lyot stop) and D denotes the final focal plane (with the detector). Plane D is not represented in Fig. 1.

The correct prolate spheroidal apodization is applied (in amplitude) to the wave in plane $\mathrm{A}$. In plane $\mathrm{B}$, the $\pi$ phase mask is set at the center of the telescope impulse response. The direct wave, which corresponds to an image of the pupil, and the wave diffracted by the mask interfere destructively inside the aperture in plane $\mathrm{C}$. Thanks to the properties of prolate functions and given appropriate masking in plane $\mathrm{C}$, the star is totally nulled in the monochromatic perfect case.

It is important to note that the R\&R (apodized or not) accepts central obscuration, in contrast with the FQPM/PKC or PALC. Moreover, it leaves the image outside the mask almost unaffected, without blind zones (FQPM/PKC, shaped or apodized pupil concepts (Nisenson \& Papaliolios 2001) would require rotation of the instrument) or symmetrization of the image (AIC).

The unapodized version of the Roddier \& Roddier coronagraph has been tested experimentally (Guyon et al. 1999) but with low performance, due to insufficient phase shift precision. The main drawback of this coronagraph remains its double chromatic dependence: the exact $\pi$ phase shift and optimal mask diameter are only satisfied at a single wavelength. Although great advances are being made toward achromatic phase shifting using multilayer dichroic filter technology (Riaud et al. 2001), the size chromatism problem may only be solved by adding chromatic magnification and demagnification using several lenses before the mask and between the mask and the Lyot Stop using Wynne's achromatization technique (Wynne 1979; Roddier et al. 1980). These chromatic effects for a PARRC are represented in Fig. 2 in the worst case of uncompensated double chromatism, and for size chromatism alone.

The chromatism effects can be illustrated by a simple reasoning at the center of the coronagraphic pupil, in plane $\mathrm{C}$. 
Phase \& Size Chromatism
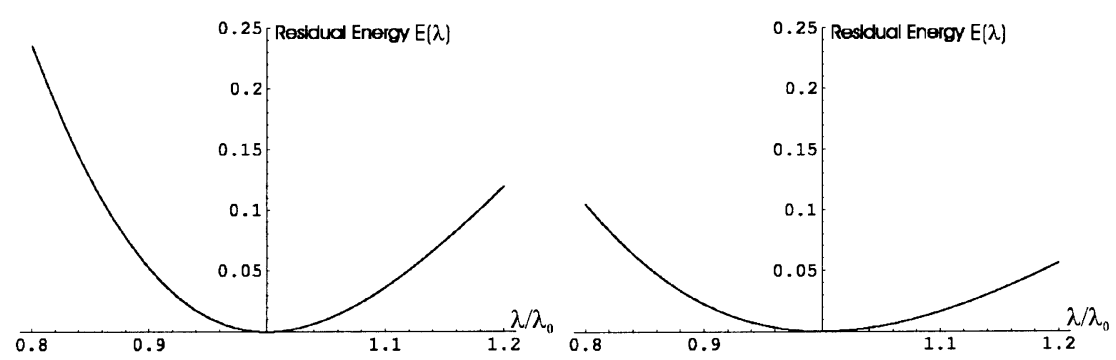

Fig. 2. This figure illustrates the deviation from the perfect monochromatic case for the PARRC. The figures represent the residual star energy integrated inside the exit pupil and normalized to the apodizer throughput $T$, as a function of the wavelength $E(\lambda)=\frac{1}{T} \int_{0}^{D / 2} 2 \pi r\left|\Psi_{\mathrm{C}}(r, \lambda)\right|^{2} \mathrm{~d} r$. as a function of the wavelength for Prolate Apodized Roddier \& Roddier Coronagraph. The left figure represents $E(\lambda)$ considering a simple reflection $\pi$ phase mask (double chromatism dependence). Right figure: even with a perfect achromatic phase shift, the effet of size chromatism alone severely limits the performance of the coronagraph. Note that the residual energy is exactly zero at the central wavelength.
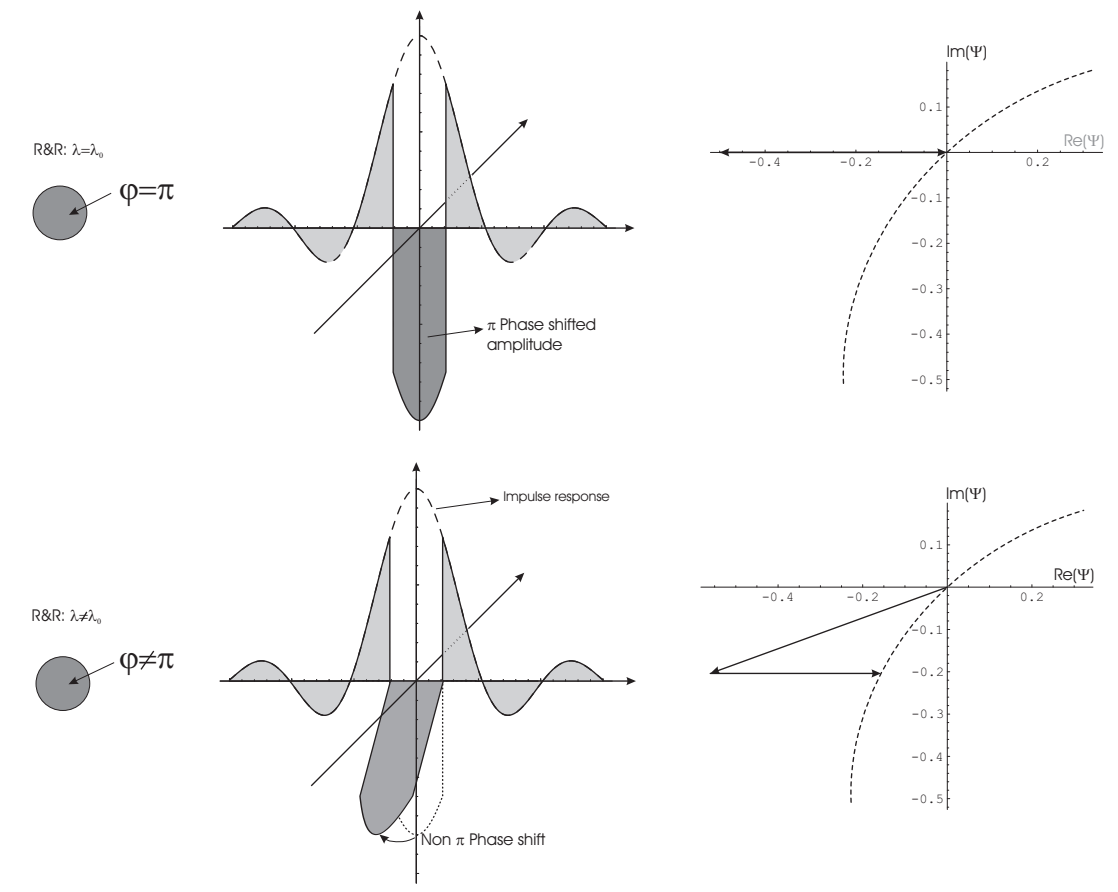

Fig. 3. Schematic illustration of the double chromatism for R\&R (or PARRC). Top: at $\lambda=$ $\lambda_{0}$ the phase shift is exactly $\pi$. In particular, at the center of the pupil in plane $\mathrm{C}$, the wave amplitude exactly zero: the integral of the amplitude inside and outside the mask (in plane B),represented in grey tints, are equal and opposite (center). These two integrals are represented by 2 opposite vectors in the complex plane (right). Center: at $\lambda \neq \lambda_{0}$ the phase shift is not exactly $\pi$ (for a reflection phase mask for example) and the impulse response size is magnified by the factor $\lambda_{0} / \lambda$. The corresponding vectorial addition is no longer null (right), and describes the dotted line curve over the bandwidth. Bottom: to obtain an achromatic nulling at the center of the pupil, a third vector is necessary in the complex plane to obtain again a zero complex amplitude (right). This third vector can be produced using a second doughnut-shaped phase mask. A dual-zone phase mask with two non $\pi$ phase shifts is then needed.

The amplitude at the center of the pupil in plane $\mathrm{C}$ is equal to the integral of the complex amplitude in plane B (Fourier integral theorem). This integral over the whole plane B can be split into two integrals: inside and outside the mask area.

The complex amplitude $\boldsymbol{\Psi}$ at the center of the coronagraphic pupil (plane $\mathrm{C}$ ) is then the complex addition of the two vectors $\boldsymbol{v}_{1}=v_{1} \mathrm{e}^{i \varphi_{1}}$, representing the integrated field within the phase shifted zone, and $\boldsymbol{v}_{2}=v_{2} \mathrm{e}^{i \varphi_{2}}$ representing the integrated field outside this zone. For perfect nulling at the center of the pupil, $\boldsymbol{v}_{1}=\boldsymbol{v}_{2} \mathrm{e}^{l \pi}$, and the two vectors are real, as illustrated in Fig. 3 (top).

The chromatic dependence of this scheme can easily be demonstrated by this representation. When the wavelength decreases slightly, the Airy disk shrinks so that more energy is 
concentrated in the inner zone, making $v_{1}$ grow and $v_{2}$ decrease. Also, unless a sophisticated achromatic phase shifter is used, the phase between the vectors is no longer 180 degrees. For example, in the case of a reflective phase mask, optimized at the wavelength $\lambda_{0}$ with a step size $z=\lambda_{0} / 4$, the phase shift between the two waves at the wavelength $\lambda$ is $\varphi=\pi \lambda_{0} / \lambda$, and the vector $\boldsymbol{v}_{1}$ rotates in the complex plane (Fig. 3). The resulting vector $\boldsymbol{\Psi}=\boldsymbol{v}_{1}+\boldsymbol{v}_{2}$ is now finite, i.e. $\mathrm{d}|\boldsymbol{\Psi}| / \mathrm{d} \lambda \neq 0$

This vectorial addition reasoning holds for a single point in the pupil, and the Fourier area theorem makes it particularly easy to perform this sum at the center of the pupil. However, knowing the profile of the Fourier transform of each zone of the phase-masked image, it is easy to generalize this treatment to every point in the pupil.

\subsection{Dual-zone phase mask}

The above reasoning for the $R \& R$ Phase Mask induces the necessity of a third vector $v_{3}$, that would be able to reduce to zero (or approximately zero) the complex amplitude $\boldsymbol{\Psi}$ at the center of the pupil for a range of wavelengths.

For that, let us consider a second, doughnut-shaped phase mask, surrounding the original R\&R Phase disk, as illustrated in Fig. 3. Two different phase shifts $\varphi_{1}$ and $\varphi_{2}$ are attributed to this dual-zone phase mask. The complex amplitude at the center of the coronagraphic pupil now appears as the summation of three complex components: $\boldsymbol{\Psi}=\boldsymbol{v}_{1}+\boldsymbol{v}_{2}+\boldsymbol{v}_{3}$.

An infinite number of solutions may be found satisfying $\Psi=\boldsymbol{v}_{1}+\boldsymbol{v}_{2}+\boldsymbol{v}_{3}=0$, but one may also hope to find at least one solution for which $\mathrm{d} \Psi / \mathrm{d} \lambda=0$. This would indicate the presence of a zone within which $\Psi \approx 0$ for a finite wavelength range.

Let us first illustrate this proposal in the simple case of a raw unapodized aperture. We consider a plane wave of complex amplitude unity, arriving at the telescope aperture. In the focal plane, the complex amplitude of the Airy pattern is:

$\mathrm{A}(r)=D \frac{J_{1}(\pi D r / \lambda f)}{2 r}$,

where $D$ is the telescope diameter, $f$ the focal length, $\lambda$ the wavelength and $r$ the position. At the center of the re-image pupil, the complex amplitude is unity, using the Fourier integral theorem:

$\frac{1}{\lambda f} \int_{0}^{\infty} 2 \pi r \mathrm{~A}(r) \mathrm{d} r=1$

with the Fourier Optics scaling factor $1 / \lambda f$ (Goodman 1996). The integral over each zone finds an analytical expression, using the derivation property of Bessel functions $\mathrm{d} J_{0}(r) / \mathrm{d} r=-J_{1}(r)$ :

$\operatorname{Int}(d, \lambda)=\frac{1}{\lambda f} \int_{0}^{d / 2} 2 \pi \xi \mathrm{A}(\xi) \mathrm{d} \xi=1-J_{0}\left(\frac{\pi D d}{2 \lambda f}\right)$.

The modulus of the three vectors $\boldsymbol{v}_{1}, \boldsymbol{v}_{2}, \boldsymbol{v}_{3}$ corresponds to the integrals of the complex amplitude over the three zones:

$v_{1}=\operatorname{Int}\left(d_{1}, \lambda\right)$

$v_{2}=\operatorname{Int}\left(d_{2}, \lambda\right)-\operatorname{Int}\left(d_{1}, \lambda\right)$,

$v_{3}=1-\left(v_{1}+v_{2}\right)$

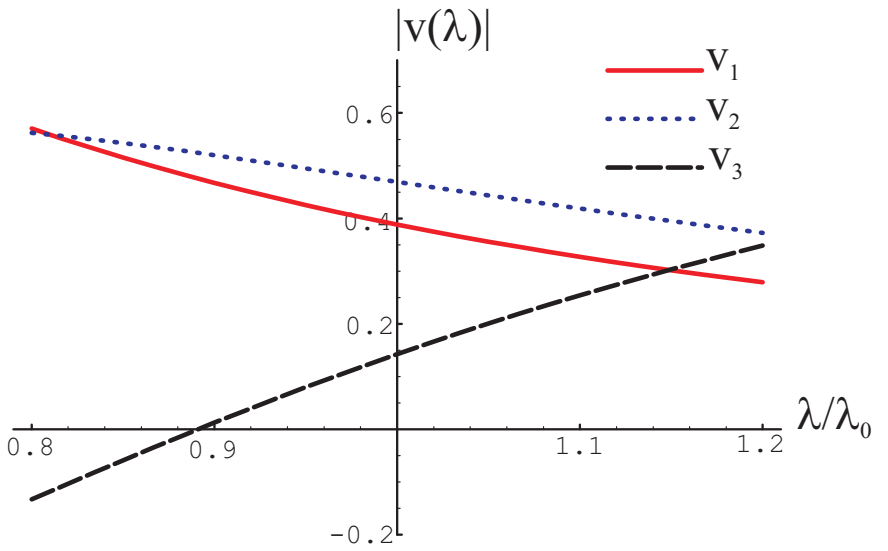

Fig. 4. Illustration of the modulus of the three vectors $\boldsymbol{v}_{1}, \boldsymbol{v}_{2}, \boldsymbol{v}_{3}$ for a variation wavelength $\lambda / \lambda_{0}$ across the bandwidth, corresponding to Eq. (4).

where $d_{1}$ and $d_{2}$ are the diameters of the inner and outer zone of the mask, expressed in units of the resel size $\lambda_{0} f / D$ at the central wavelength $\lambda_{0}$. Theses integrals are illustrated in Fig. 4 for a bandwidth of $40 \%$.

The phase shift in each zone must be calculated as a function of the wavelength. Assuming a reflective phase mask with steps $z_{1}$ and $z_{2}$ expressed in units of $\lambda_{0}$, the phases are given by: $\varphi_{1}=4 \pi z_{1} \lambda_{0} / \lambda, \varphi_{2}=4 \pi z_{2} \lambda_{0} / \lambda$ and $\varphi_{3}=0$. The complex amplitude at the center of the pupil $\Psi$ :

$\boldsymbol{\Psi}=\boldsymbol{v}_{1}+\boldsymbol{v}_{2}+\boldsymbol{v}_{3}=v_{1} \mathrm{e}^{i \varphi_{1}}+v_{2} \mathrm{e}^{l \varphi_{2}}+v_{3} \mathrm{e}^{l \varphi_{3}}$

finds then an analytical expression using Eq. (4):

$\boldsymbol{\Psi}=\mathrm{e}^{i \varphi_{1}}+\left[\mathrm{e}^{i \varphi_{2}}-\mathrm{e}^{i \varphi_{1}}\right] J_{0}\left(\frac{\pi D d_{1}}{2 \lambda f}\right)+\left[1-\mathrm{e}^{i \varphi_{2}}\right] J_{0}\left(\frac{\pi D d_{2}}{2 \lambda f}\right)$.

The mean polychromatic intensity at the center of the pupil, for the bandwidth $\Delta \lambda$ centered at the wavelength $\lambda_{0}$ can be used as a criterion to search for an achromatic solution (at the center of the pupil):

$I_{0}=\frac{1}{\Delta \lambda} \int_{\lambda_{0}-\Delta \lambda / 2}^{\lambda_{0}+\Delta \lambda / 2}|\Psi|^{2} \mathrm{~d} \lambda$.

A numerical conjugate gradient method has been used to optimize the four parameters $d_{1}, d_{2}, z_{1}, z_{2}$. The representation of Fig. 3 shows that the mask must have dimensions comparable to the Airy core to obtain a suitable three-vectors addition. This information is very useful in choosing the starting values of $d_{1}$ and $d_{2}$ for the optimization, and limits the range of investigation to discriminate local minima. Moreover, the analytical expression (Eq. (6)) is easily implemented and provides efficient calculations.

The optimal set of solutions depends on the bandwidth and this point will be specifically discussed below. In this simple case, we have tested the three bandwidths 20\%, 30\% and $40 \%$ : the mask dimensions remain within a single group in the $\left(d_{1}, d_{2}\right)$ plot, Fig. 5 (left). However, four main groups of solutions can be identified for the phase steps in Fig. 5 (right), that we denote S1, S2, S3 and S4. We have represented in Fig. 6 the intensity $I_{0}$ for a typical fixed solution $\left(d_{1}, d_{2}\right)$ (chosen in the 

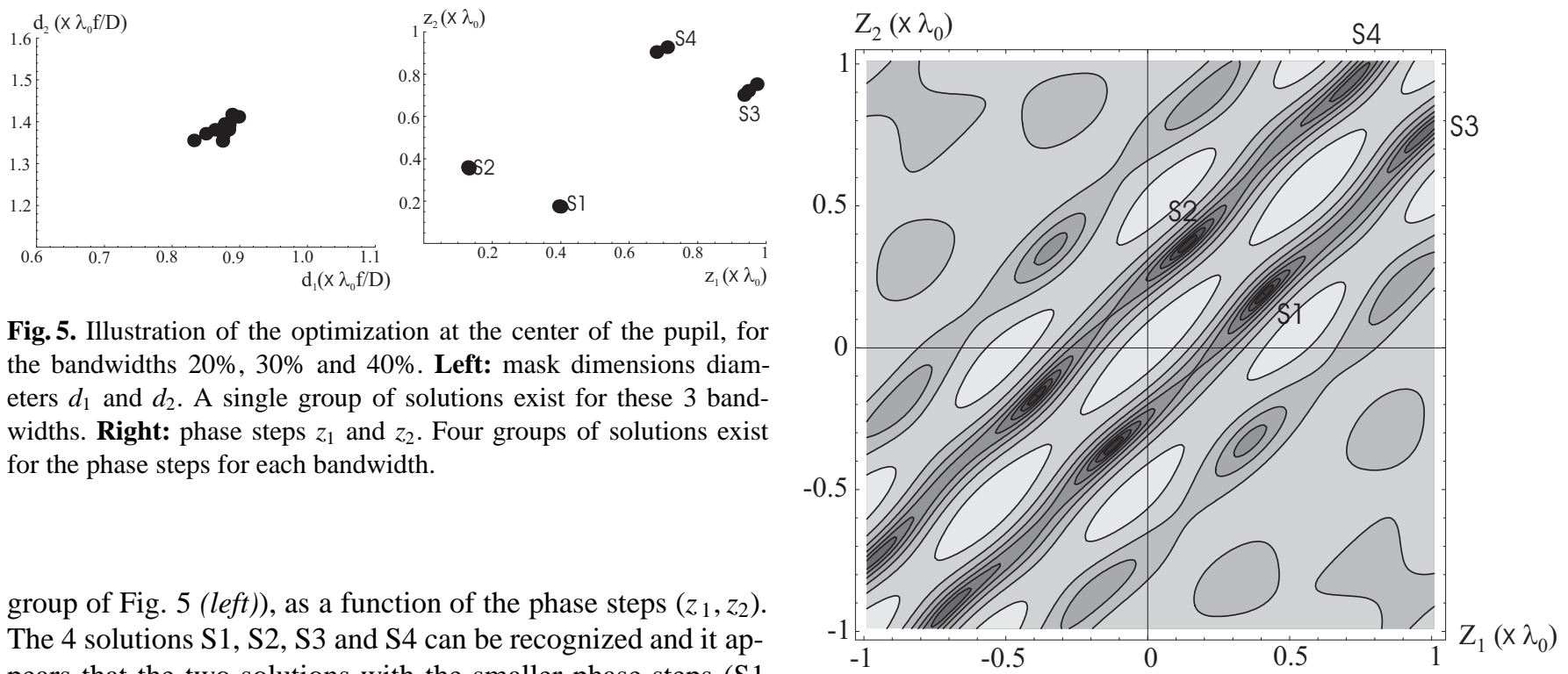

group of Fig. 5 (left)), as a function of the phase steps $\left(z_{1}, z_{2}\right)$. The 4 solutions S1, S2, S3 and S4 can be recognized and it appears that the two solutions with the smaller phase steps ( $\mathrm{S} 1$ and S2) are more interesting: they correspond to a deeper level on the contour representation of Fig. 6. This is not surprising since for large phase steps $z$, the chromatism of the phase shift is greater. It is therefore more difficult to compensate over large bandwidths.

For these optimized solutions, the resulting complex amplitude $\Psi$ at the center of the pupil traces a $\gamma$-like curve in the complex plane, as $\lambda$ varies over the bandwidth. Each point on this curve corresponds to the three-vector addition described qualitatively in Fig. 3 and illustrated for the parameters of solution (S2) in Fig. 7. The wave amplitude is folded around zero in the complex plane so that the intensity remains close to zero for a large range of wavelengths.

For the best of these four solutions (S2) we have summarized the results in Fig. 8 in terms of $\Psi$, the complex amplitude at the center of the pupil corresponding to the two-vector addition for R\&R described in Fig. 3 and to the three-vector addition for DZPM. The intensity at the center the pupil is compared to the original unapodized Roddier \& Roddier technique over the bandwidth. Near perfect nulling is achieved at two points and less than $0.5 \%$ is reached over most of the band.

Although this analysis only holds for a single point in the pupil, it is a good introduction the problem of full-pupil optimization, described in the following section.

\section{Optimization over the entire pupil}

\subsection{Formalism}

In this section we give the formalism of the dual-zone coronagraph for a circular aperture, based on the formalism developed for prolate apodized circular apertures and coronagraphy (Soummer et al. 2003). Thanks to the geometry of the pupil, apodization and phase masks, the problem presents a radial symmetry: a two-dimensional function $f(\boldsymbol{r})$ can be represented by its radial cut $f(r)$, where $r=\sqrt{x^{2}+y^{2}}$ is the modulus of the position vector $\boldsymbol{r}=(x, y)$. The wave propagation between each plane A, B, C, D involved in the coronagraph can be written as a scaled Fourier Transform (FT) (Goodman 1996). The FT of

Fig. 6. Illustration of the existence of several solutions for the phase steps $z_{1}$ and $z_{2}$ for a typical solution of mask diameters $d_{1}=$ $0.88 \lambda_{0} f / D$ and $d_{2}=1.40 \lambda_{0} f / D$ (see Fig.5). The figure represents the intensity $I_{0}$ as a function of the phase steps $z_{1}$ and $z_{2}$. The contour scaling is logarithmic with 10 equally-spaced contours between $10^{-3}$ and 1 (dark shading corresponds to lower values). The best solutions correspond to the smallest phase step values (S1 and S2). The other solutions S3, S4, etc. have a lower efficiency (the figure is centrosymmetrical and identical solutions exist with negative phase steps). The points of Fig. 5 correspond to the four holes of the figure (for positive values of $z_{1}$ and $z_{2}$ ). The solutions denoted S1 and S2 correspond to a lower intensity (clearly visible on the contour plot). This computation is made for $40 \%$ bandwidth.

a radial function is also a radial function, whose radial cut can be expressed using the Hankel Transform (HT):

$\widehat{\Psi}_{\mathrm{A}}(r)=\int_{0}^{\infty} 2 \pi \rho \Psi_{\mathrm{A}}(\rho) J_{0}(2 \pi \rho r) \mathrm{d} \rho$

We consider a plane wave arriving at the circular aperture of diameter $D$ apodized by a radial function $\boldsymbol{\Phi}(r)$ (eventually $\boldsymbol{\Phi}$ can be complex in this paper). The complex amplitude at the aperture is then $\Psi_{\mathrm{A}}(\boldsymbol{r})=\Pi\left(\frac{r}{D}\right) \boldsymbol{\Phi}(\boldsymbol{r})$, where $\Pi(r)=1$ for $|r| \leq$ $1 / 2$ and 0 otherwise.

In the focal plane, the Dual-Zone Phase Mask (DZPM) size is proportional to the size of the diffraction pattern for the central wavelength of the band $\lambda_{0}$. The DZPM inner diameter $d_{1}$ and outer diameter $d_{2}$ are written as:

$d_{1}=\alpha_{1} \frac{\lambda_{0} f}{D}=a_{1} \lambda_{0} f$
$d_{2}=\alpha_{2} \frac{\lambda_{0} f}{D}=a_{2} \lambda_{0} f$

where we use the parameters $a_{1}$ and $a_{2}$ to express the diameters in units of $1 / D$, for convenience in the following equations and for homogeneity with the previous paper notation 

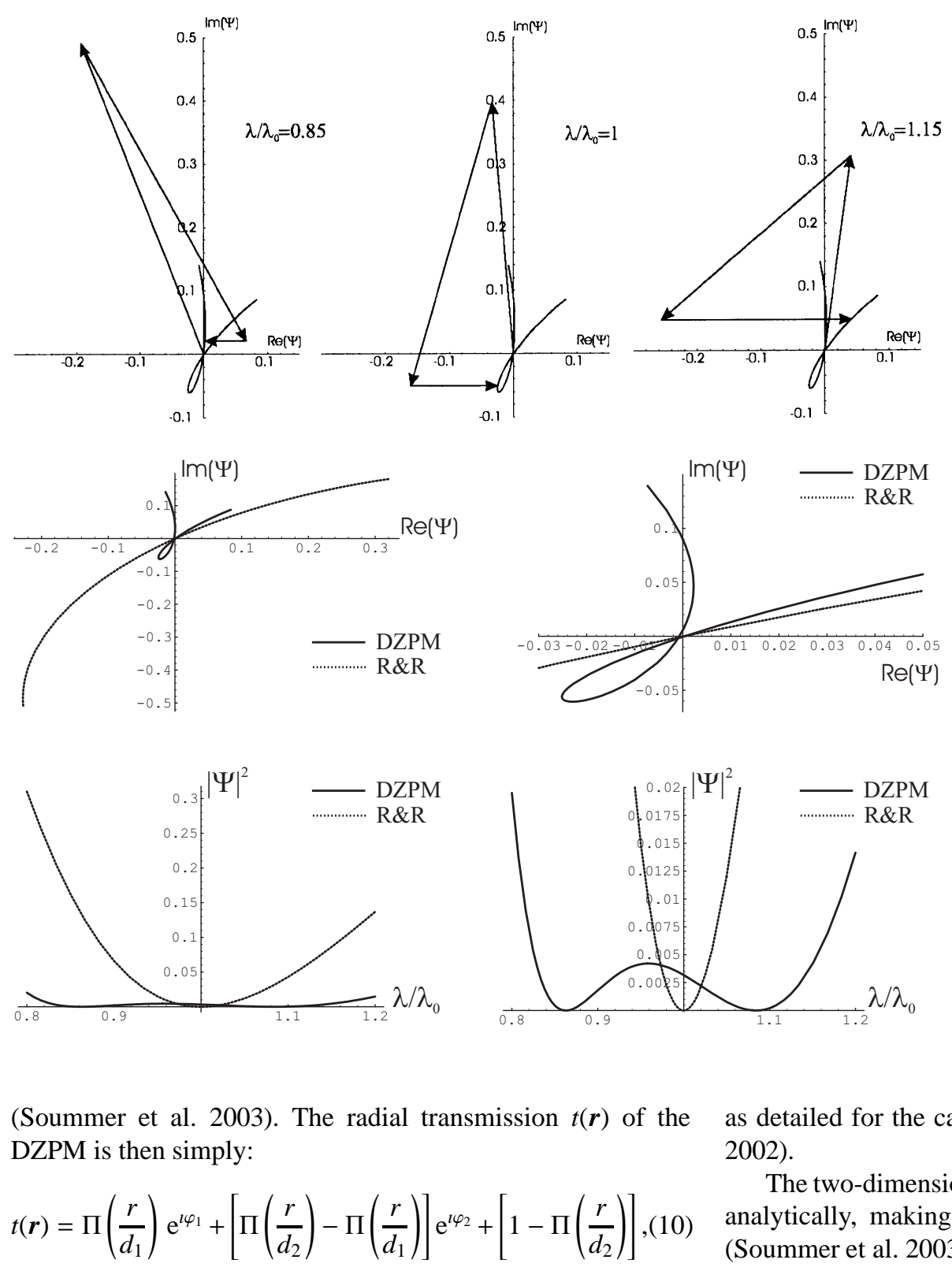

(Soummer et al. 2003). The radial transmission $t(\boldsymbol{r})$ of the DZPM is then simply:

$t(\boldsymbol{r})=\Pi\left(\frac{r}{d_{1}}\right) \mathrm{e}^{i \varphi_{1}}+\left[\Pi\left(\frac{r}{d_{2}}\right)-\Pi\left(\frac{r}{d_{1}}\right)\right] \mathrm{e}^{i \varphi_{2}}+\left[1-\Pi\left(\frac{r}{d_{2}}\right)\right]$,

or:

$t(\boldsymbol{r})=1-\Pi\left(\frac{r}{d_{1}}\right)\left[\mathrm{e}^{i \varphi_{2}}-\mathrm{e}^{i \varphi_{1}}\right]-\Pi\left(\frac{r}{d_{2}}\right)\left[1-\mathrm{e}^{i \varphi_{2}}\right]$.

The wave amplitude after the mask is written:

$\Psi_{\mathrm{B}}(\boldsymbol{r}, \lambda)=\frac{1}{\imath \lambda f} \widehat{\Psi}_{\mathrm{A}}\left(\frac{\boldsymbol{r}}{\lambda f}\right) \times \mathrm{t}(\boldsymbol{r})$

The analytical computation here is analog to the PARRC formalism, but with two coronagraphic terms instead of a unique coronagraphic term (see Eq. (6) of Soummer et al. 2003). The chromatic complex amplitude in the pupil plane $\mathrm{C}$ is obtained by performing a scaled FT of Eq. (12), to obtain:

$$
\begin{aligned}
\Psi_{\mathrm{C}}^{-}(\boldsymbol{r}, \lambda)= & \Psi_{\mathrm{A}}(\boldsymbol{r}) \\
& -\Psi_{\mathrm{A}}(\boldsymbol{r}) * \frac{\lambda_{0}}{\lambda} \frac{a_{1}}{2 r} J_{1}\left(\pi a_{1} r \frac{\lambda_{0}}{\lambda}\right)\left[\mathrm{e}^{i \varphi_{2}}-\mathrm{e}^{i \varphi_{1}}\right] \\
& -\Psi_{\mathrm{A}}(\boldsymbol{r}) * \frac{\lambda_{0}}{\lambda} \frac{a_{2}}{2 r} J_{1}\left(\pi a_{2} r \frac{\lambda_{0}}{\lambda}\right)\left[1-\mathrm{e}^{i \varphi_{2}}\right],
\end{aligned}
$$

Fig. 7. Illustration of the three vector addition in the complex plane, for three wavelengths $\lambda / \lambda_{0}=0.85, \lambda / \lambda_{0}=1, \lambda / \lambda_{0}=$ 1.15 , and parameters $d_{1}, d_{2}, z_{1}, z_{2}$ of solution $\mathrm{S} 2$. The resulting vector describes the $\gamma$-like curve as the wavelength varies across the bandwidth.

Fig. 8. Results of the optimization at the center of the pupil. Top: representation in the complex plane of the complex amplitude at the center of the pupil. The complex amplitude is folded with a $\gamma$-like curve and remains close to zero. A comparison is made at the same scale with $R \& R$ (left) and zoomed (right). Bottom: residual intensity at the center of the pupil as a function of the wavelength, compared to the $\mathrm{R} \& \mathrm{R}$ coronagraph (left) and zoomed (right). The intensity is normalized to 1 for the case without coronagraph. Almost perfect nulling is obtained for two wavelengths, and the average intensity is below $0.5 \%$ over the bandwidth. The comparison with the $\mathrm{R} \& \mathrm{R}$ coronagraph, without chromatism correction, shows a great improvement with the DZPM technique. as detailed for the case of a simple mask (Aime \& Soummer 2002).

The two-dimensional convolution product can be computed analytically, making use of the chromatic kernel $K_{0}(\xi, r, a)$ (Soummer et al. 2003). Equation (13) then becomes:

$$
\begin{aligned}
\Psi_{\mathrm{C}}^{-}(\boldsymbol{r}, \lambda)= & \Psi_{\mathrm{A}}(\boldsymbol{r}) \\
& -(2 \pi)^{2} \int_{0}^{\frac{D}{2}} \xi \boldsymbol{\Phi}(\xi) K_{0}\left(\xi, r, a_{1} \frac{\lambda_{0}}{\lambda}\right) \mathrm{d} \xi\left[\mathrm{e}^{l \varphi_{2}}-\mathrm{e}^{l \varphi_{1}}\right] \\
& -(2 \pi)^{2} \int_{0}^{\frac{D}{2}} \xi \boldsymbol{\Phi}(\xi) K_{0}\left(\xi, r, a_{2} \frac{\lambda_{0}}{\lambda}\right) \mathrm{d} \xi\left[1-\mathrm{e}^{l \varphi_{2}}\right],
\end{aligned}
$$

recalling the kernel expression:

$K_{0}(\xi, r, a)=\frac{a r J_{0}(a \pi \xi) J_{1}(a \pi r)-a \xi J_{0}(a \pi r) J_{1}(a \pi \xi)}{4 \pi\left(r^{2}-\xi^{2}\right)}$,

and its limit for $r=\xi$ :

$K_{0}(\xi, \xi, a)=\frac{1}{8} a^{2}\left[J_{0}^{2}(a \pi \xi)+J_{1}^{2}(a \pi \xi)\right]$.

A Lyot Stop is set to remove the light diffracted outside the aperture:

$\Psi_{\mathrm{C}}^{+}(\boldsymbol{r}, \lambda)=\Psi_{\mathrm{C}}^{-}(\boldsymbol{r}, \lambda) \times \Pi\left(\frac{r}{D}\right)$. 
A Lyot Stop diameter reduction can be eventually optimized, but with entrance pupil apodization, this point is no longer essential (Aime et al. 2001). Finally, an expression of the residual chromatic focal intensity can be obtained by applying a scaled Hankel Transform to Eq. (17).

The coronagraphic effect can be analyzed in the pupil plane $\mathrm{C}$, as in the previous studies: for a classical $\mathrm{R} \& \mathrm{R}$ (or Lyot) coronagraph, the amplitude in plane $\mathrm{C}$ is written as the subtraction of two waves: the direct wave (the pupil complex amplitude) and the wave diffracted by the mask. For the DZPM, Eq. (13) is analogous to Eq. (6) of Soummer et al. (2003), but for the fact that there are three different complex profiles to match in the pupil plane $\mathrm{C}$.

An entrance pupil apodization is also necessary for the DZPM, as for R\&R or Lyot masks (Aime \& Soummer 2002) since the smooth diffracted amplitudes cannot perfectly match a flat entrance pupil amplitude. However, we may consider here a complex apodization, which can be obtained by adding radially symmetric aberrations (defocus and any order of spherical aberration) to the wavefront in the entrance pupil .

The reasoning we made at the center of the pupil must hold in each point of the pupil, as suggested by Eq. (13): the two subtracted wavefronts, expressed by the convolution products, are balanced by the two complex coefficients $\mathrm{e}^{i \varphi_{2}}-\mathrm{e}^{i \varphi_{1}}$ and $1-\mathrm{e}^{i \varphi_{2}}$, with the effect of rotating the wavefronts in the complex plane and modifying their amplitude. This triple complex wavefront addition is represented in Fig. 9 inside the aperture (the two subtractive wavefronts extend beyond the pupil aperture). When the wavelength varies over the band, their shape, rotation angle and amplitude are modified (modification of the kernel of Eq. (14) and of the two complex coefficients). The goal is to obtain a compensation of these effects so that the addition of the two diffracted wavefronts remains approximately opposite to the entrance pupil amplitude over the bandpass (Fig. 9).

\subsection{Optimization}

The optimal configuration that fits the above reasoning can be obtained by minimizing the mean polychromatic residual energy inside the aperture (the Lyot Stop). This criterion can be written:

$R=\frac{1}{T \Delta \lambda} \int_{\lambda_{0}-\Delta \lambda / 2}^{\lambda_{0}+\Delta \lambda / 2} \int_{0}^{D / 2} 2 \pi r\left|\Psi_{\mathrm{C}}^{-}(r, \lambda)\right|^{2} \mathrm{~d} r \mathrm{~d} \lambda$,

where the normalization by $T$ corresponds to the throughput of the apodization alone:

$T=\int_{0}^{D / 2} 2 \pi r|\boldsymbol{\Phi}(\boldsymbol{r})|^{2} \mathrm{~d} r$.

Note that we assume here a white object and a square bandwidth for simplicity.

\subsubsection{Results without entrance pupil apodization}

We first analyse the case of the DZPM technique for a raw unapodized aperture: the problem depends on the four parameters $d_{1}, d_{2}, z_{1}, z_{2}$. Several solutions exist; $\mathrm{S} 1$ and $\mathrm{S} 2$ can still

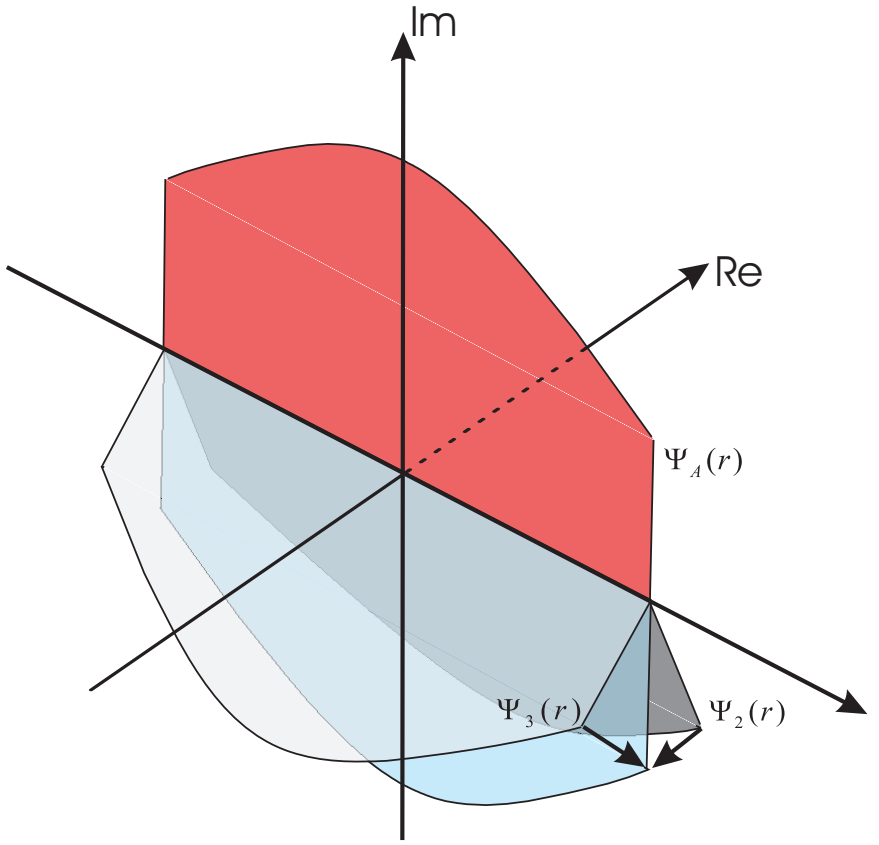

Fig. 9. Heuristic illustration of Eq. (13). With the DZPM, there are three complex profiles to match: the apodized pupil $\Psi_{\mathrm{A}}(r)$ and the two complex wavefronts $\Psi_{2}(r)$ and $\Psi_{3}(r)$. The sum of these two complex wavefront is expected to be real and to match the opposite of the pupil complex amplitude. Note that the two wavefronts $\Psi_{2}(r)$ and $\Psi_{3}(r)$ extend beyond the pupil aperture (not represented here). When the wavelength varies across the bandwidth, we expect to obtain a compensation of the shape modification and rotation for $\Psi_{2}(r)$ and $\Psi_{3}(r)$ to obtain an achromatic coronagraphic effect.

be identified from their phase steps (the values are similar to the results for optimization at the center of the pupil), but with different mask sizes. In Fig. 10, we have represented the criterion $R$ for $\mathrm{S} 1$ and $\mathrm{S} 2$, compared to the unapodized $\mathrm{R} \& \mathrm{R}$ technique. In this case, without apodization, S2 shows a significant improvement over the $\mathrm{R} \& \mathrm{R}$ technique and $\mathrm{S} 1$ is equivalent to $R \& R$. This improvement with $S 2$ can be very interesting in cases where the pupil is not available for apodization, such as for the coronagraphic mode in the NGST/MIRI instrument.

\subsubsection{Results with entrance pupil apodization}

Numerous possibilities have been tested. First, a pure phase apodization was tried unsuccessfully and abandoned. The pure amplitude apodization gives a great improvement, as expected. However, prolate apodizations that are the eigenfunctions of the kernels of Eq. (14) cannot be used for the DZPM technique: the same apodization function $\Phi(r)$ appears in the two integrals of Eq. (14) and it cannot simultaneously be the eigenfunction for the two different kernels.

We have instead used a polynomial apodization function. It appeared from ours tests that a fourth order polynomial is sufficient. For the additional phase apodization, we tested the influence of several radial orders, but only defocus plays a role (and only for S2). The complex apodization to optimize is then written as:

$\boldsymbol{\Phi}(\boldsymbol{r})=\left(1+\alpha r^{2}+\beta r^{4}\right) \mathrm{e}^{\frac{2 \imath \pi}{\lambda}\left(\chi r^{2}\right)}$. 


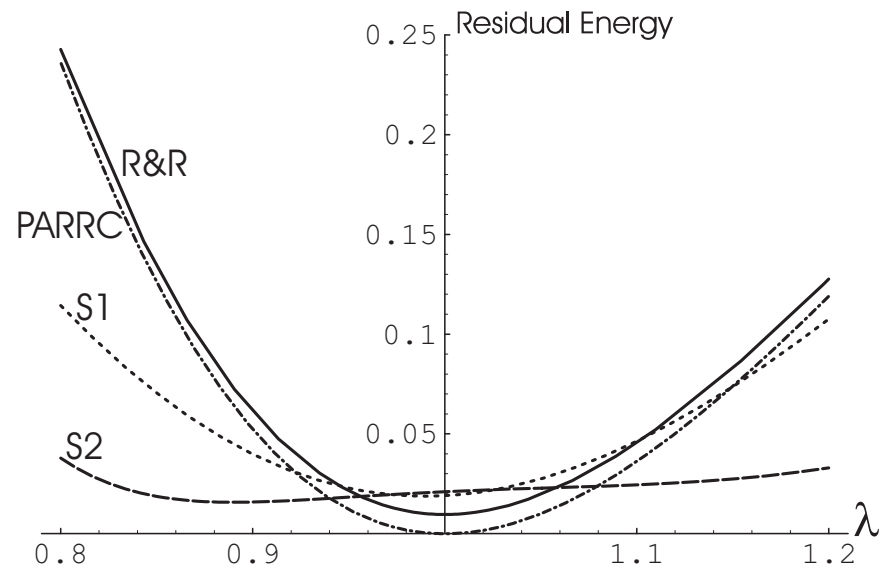

Fig. 10. Illustration of the residual integrated energy over the entire pupil, for the full pupil optimization without apodization. The comparison is made for unapodized and uncompensated R\&R (full line), Prolate Apodized R\&R (dash dotted), S1 (dotted line) and S2 (dashel line). Respectively, the mean chromatic residual energies are $6.8 \%$, $5.0 \%$ and $2.2 \%$. S2 shows a significant improvement over R\&R. An optimization over a smaller bandwidth increases the effect. This result is interesting for instruments for which the pupil cannot be apodized (e.g. NGST).
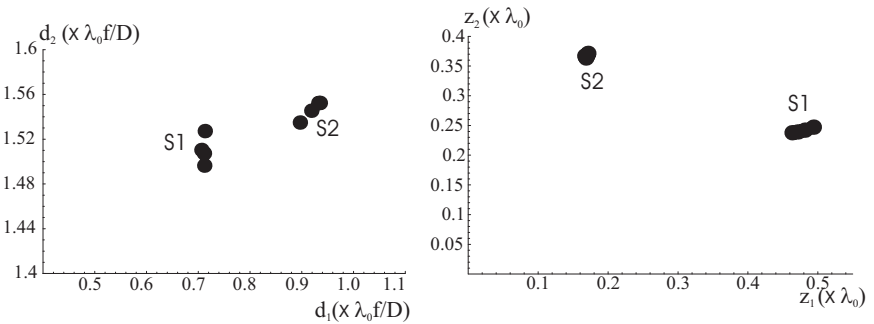

Fig. 11. Results of the optimization in the entire pupil, including complex apodization (amplitude + slight defocus) for bandwidths $40 \%$, $30 \%, 20 \%$ and $10 \%$. The representation is similar to in Fig. 5. The full pupil optimization leads to a differentiation of the mask dimensions for each of the phase steps solutions. Only the two solutions S1 and $\mathrm{S} 2$ are represented. Note that only $\mathrm{S} 2$ requires a slight defocus.

The optimization problem now includes 7 parameters $\left(d_{1}, d_{2}, z_{1}, z_{2}, \alpha, \beta, \chi\right)$. As in Fig. 5, we still find four main groups of solutions for the phase steps $\left(z_{1}, z_{2}\right)$. However, with apodization, a single group no longer exists in the $\left(d_{1}, d_{2}\right)$ plot. A group of mask dimensions $\left(d_{1}, d_{2}\right)$ corresponds to each of the four phase groups of solutions $\left(z_{1}, z_{2}\right)$. The two solutions $\mathrm{S} 1$ and $\mathrm{S} 2$ can still be identified and are represented in Fig. 11. Note that the values also depend on the bandwidth (this dependence will be analysed in the discussion). The corresponding DZPMs are represented in Fig. 12 and a typical entrance pupil apodization is given in Fig. 13.

The best solution, that we will consider in the following, is S2, especially when complex apodization is used. A slight defocus (simply defocusing the mask from the focal plane) provides for example a gain of 3 on the integrated energy for a bandwidth of $40 \%$ and a gain of 15 for a bandwidth of $20 \%$. This defocus is very small and corresponds typically to $\lambda / 20$ at the edge of the entrance pupil.

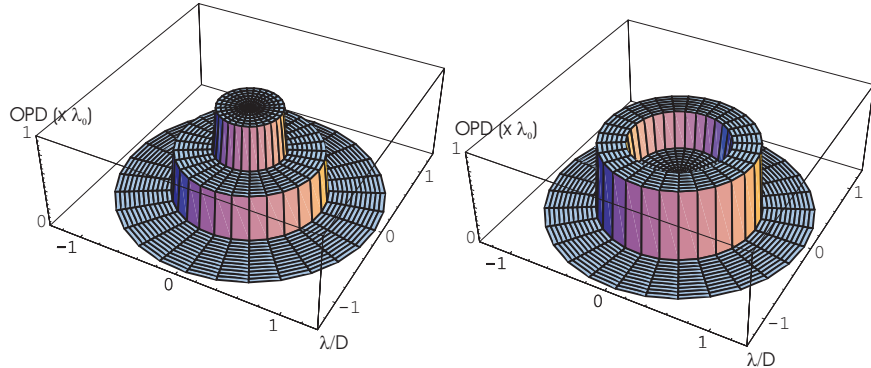

Fig. 12. Representation reflection DZPMs for S1(left) and S2(right) with $40 \%$ bandwidth. the vertical axis represents the Optical Path Difference at the wavelength $\lambda_{0}$. The horizontal extension of the plots corresponds to the dimension of the core of the diffraction pattern.

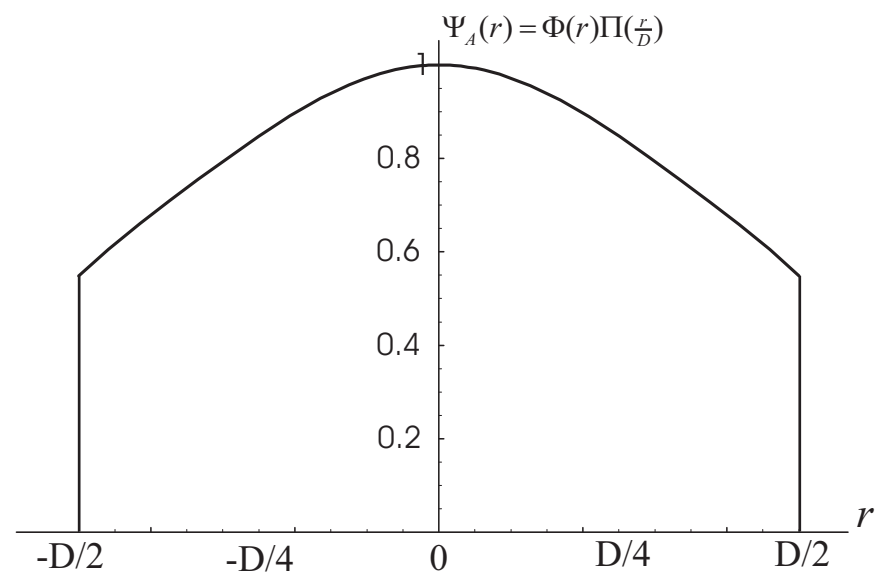

Fig. 13. Radial cut of the normalized amplitude at the entrance pupil $\Psi_{\mathrm{A}}(r)=\Phi(r) \Pi(r / D)$ (dimensionless) for S2 and $40 \%$ bandwidth. The apodizations obtained for other bandwidths are very similar.

We have represented in Fig. 14 the normalized residual energy $R$ (bandwidth of 40\%), as a function of the phase steps $z_{1}$ and $z_{2}$; the other parameters are constant and correspond to S2 values. The comparison with Fig. 6 shows that the general aspect is similar, but with a clearly identifiable global minimum. These precautions and two dimensional representations guarantee that the solution does not correspond to a local minimum.

Another interesting representation is to consider again the wave amplitude in the complex plane, at different positions in the coronagraphic pupil. The complex amplitude still describes a $\gamma$-like folded curve, at each point of the pupil. The evolution is given in Fig. 15 for a $40 \%$ bandwidth and several positions along a radial cut of the pupil.

The numerical values of the optimal parameters and summary of the performances for the best solutions are given in Table 1. Illustrations of the results for several wavelengths are given in Fig. 16. The normalized energy criterion $(R)$ is represented in Fig. 17 and can be compared to the uncorrected PARRC corresponding curve (Fig. 2): a very high improvement is brought by the double zone approach. The entrance pupil apodization has a throughput (roughly 60\%) comparable to the PARRC ideal case ( $73 \%)$.

Results in the focal plane are represented in Fig. 18: we have computed the mean polychromatic residual intensity 
Table 1. Comparison of the performances for the different techniques: PARRC, DZPM without and with apodization. The normalized residual energy $R$ is normalized to the trhroughtput. The intensity levels are coronagaphic extinction ratios, i.e. the normalization is made by the maximum of the PSF without coronagraph (with apodization alone). The phase steps are given in optical path difference (OPD).

\begin{tabular}{|c|c|c|c|c|c|c|c|c|c|c|c|}
\hline Technique & $\begin{array}{l}\text { Band } \\
(\%)\end{array}$ & $\begin{array}{l}\text { Apod. } \\
T\end{array}$ & $\begin{array}{l}\text { Mask } 1 \\
a_{1}\end{array}$ & $\begin{array}{l}\text { Mask } 2 \\
a_{2}\end{array}$ & $\begin{array}{l}\text { OPD1 } \\
2 z_{1}\end{array}$ & $\begin{array}{l}\text { OPD2 } \\
2 z_{2}\end{array}$ & $\begin{array}{l}\text { Defoc. } \\
\chi\end{array}$ & $\begin{array}{l}\text { Res. Ener. } \\
R\end{array}$ & $\begin{array}{l}\text { level@ } \\
1.5 \lambda / D\end{array}$ & $\begin{array}{l}\text { level @ } \\
3 \lambda / D\end{array}$ & $\begin{array}{l}\text { level @ } \\
7 \lambda / D\end{array}$ \\
\hline Airy & $40 \%$ & None & None & None & None & None & None & 1 & $1.0 \times 10^{-2}$ & $1.1 \times 10^{-3}$ & $1.0 \times 10^{-4}$ \\
\hline $\mathrm{R} \& \mathrm{R}$ & $40 \%$ & None & $1.05 / \mathrm{D}$ & None & $1 / 2 \lambda_{0}$ & None & None & $6.8 \times 10^{-2}$ & $1.1 \times 10^{-3}$ & $1.8 \times 10^{-4}$ & $1.0 \times 10^{-5}$ \\
\hline DZPM S2 & $40 \%$ & None & $0.94 / \mathrm{D}$ & $1.42 / D$ & $0.41 \lambda_{0}$ & $0.81 \lambda_{0}$ & None & $2.2 \times 10^{-2}$ & $1.2 \times 10^{-3}$ & $1.0 \times 10^{-4}$ & $5.6 \times 10^{-6}$ \\
\hline PARRC & $40 \%$ & $72.6 \%$ & $1.06 / \mathrm{D}$ & None & $1 / 2 \lambda_{0}$ & None & None & $6.0 \times 10^{-2}$ & $5.0 \times 10^{-4}$ & $6.8 \times 10^{-5}$ & $3.7 \times 10^{-6}$ \\
\hline DZPM S1 & $40 \%$ & $41.0 \%$ & $0.71 / \mathrm{D}$ & $1.50 / D$ & $0.93 \lambda_{0}$ & $0.47 \lambda_{0}$ & None & $1.3 \times 10^{-2}$ & $7.0 \times 10^{-4}$ & $4.0 \times 10^{-5}$ & $2.0 \times 10^{-6}$ \\
\hline DZPM S2 & $40 \%$ & $59.5 \%$ & $0.88 / \mathrm{D}$ & $1.45 / D$ & $0.35 \lambda_{0}$ & $0.74 \lambda_{0}$ & None & $8.2 \times 10^{-3}$ & $4.6 \times 10^{-4}$ & $3.0 \times 10^{-5}$ & $1.7 \times 10^{-6}$ \\
\hline DZPM S2 & $40 \%$ & $57.3 \%$ & $0.90 / \mathrm{D}$ & $1.53 / D$ & $0.34 \lambda_{0}$ & $0.73 \lambda_{0}$ & -0.20 & $2.5 \times 10^{-3}$ & $4.8 \times 10^{-5}$ & $4.7 \times 10^{-6}$ & $2.3 \times 10^{-7}$ \\
\hline$R \& R$ & $20 \%$ & None & $1.05 / \mathrm{D}$ & None & $1 / 2 \lambda_{0}$ & None & None & $2.4 \times 10^{-2}$ & $1.0 \times 10^{-3}$ & $1.0 \times 10^{-4}$ & $7.0 \times 10^{-6}$ \\
\hline DZPM S2 & $20 \%$ & None & $1.03 / \mathrm{D}$ & $1.41 / D$ & $0.47 \lambda_{0}$ & $0.92 \lambda_{0}$ & None & $1.5 \times 10^{-2}$ & $1.0 \times 10^{-3}$ & $5.6 \times 10^{-5}$ & $4.4 \times 10^{-6}$ \\
\hline PARRC & $20 \%$ & $72.6 \%$ & $1.96 / \mathrm{D}$ & None & $1 / 2 \lambda_{0}$ & None & None & $1.5 \times 10^{-2}$ & $1.3 \times 10^{-4}$ & $1.5 \times 10^{-5}$ & $1.0 \times 10^{-6}$ \\
\hline DZPM S1 & $20 \%$ & $44.6 \%$ & $0.71 / \mathrm{D}$ & $1.51 / D$ & $0.96 \lambda_{0}$ & $0.48 \lambda_{0}$ & None & $3.2 \times 10^{-3}$ & $2.0 \times 10^{-4}$ & $7.0 \times 10^{-6}$ & $1.0 \times 10^{-6}$ \\
\hline DZPM S2 & $20 \%$ & $63.2 \%$ & $0.95 / \mathrm{D}$ & $1.36 / D$ & $0.40 \lambda_{0}$ & $0.77 \lambda_{0}$ & None & $5.0 \times 10^{-3}$ & $3.1 \times 10^{-4}$ & $2.5 \times 10^{-5}$ & $1.3 \times 10^{-6}$ \\
\hline DZPM S2 & $20 \%$ & $58.3 \%$ & $0.93 / \mathrm{D}$ & $1.55 / D$ & $0.34 \lambda_{0}$ & $0.74 \lambda_{0}$ & -0.22 & $3.2 \times 10^{-4}$ & $1.0 \times 10^{-5}$ & $1.0 \times 10^{-6}$ & $1.0 \times 10^{-7}$ \\
\hline
\end{tabular}

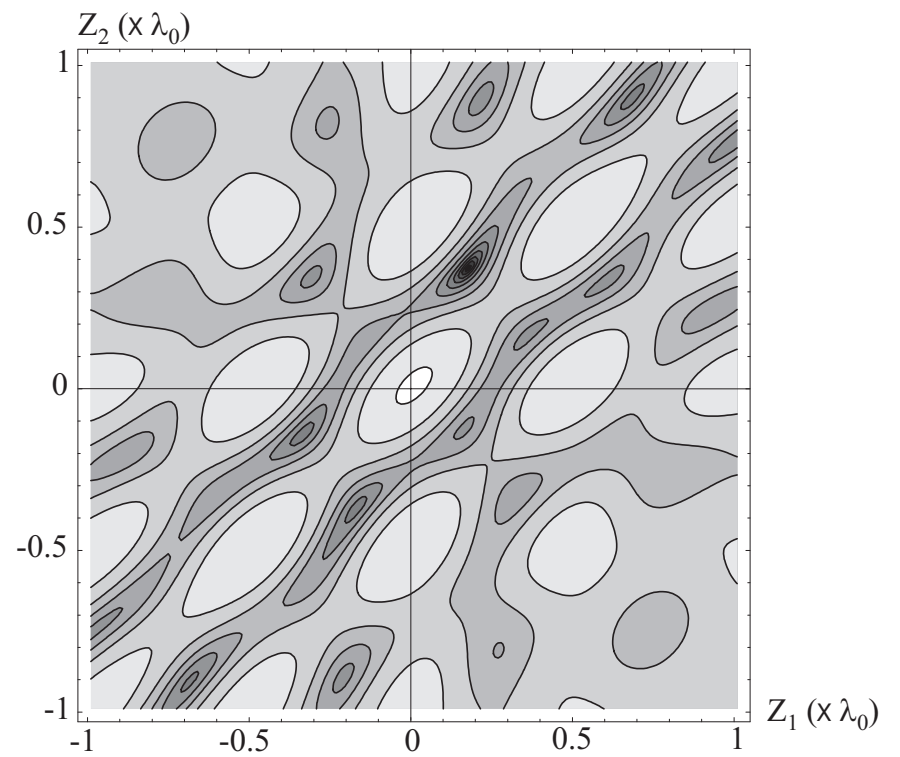

Fig. 14. Illustration of the polychromatic residual energy criterion $R$ for a bandwidth of $40 \%$ and the mask dimensions of S2, the representation is similar to Fig. 6. The contour scaling is logarithmic with 10 equally-spaced contours between $10^{-3}$ and 1 (dark shading corresponds to lower values). Complex apodization is used with a fourth order polynomial amplitude and a defocus. The presence of defocus breaks the symmetry with respect to the origin that was observed in Fig. 6 and permits a better unique global minimum (S2).

integrated over the bandwidth, and compared it to the results for PARRC and for entrance pupil apodization alone (simply removing the mask). Note that these intensities are normalized to the maximum of the intensity without the mask, so that the intensities can be read directly as coronagraphic extinction ratio.

\section{Discussion}

\subsection{Dependence on bandwidth}

In Fig. 19 we have studied the evolution of the mask dimensions $\left(d_{1}, d_{2}\right)$ and phase steps $\left(z_{1}, z_{2}\right)$, as the bandwidth decreases from $40 \%$ to zero (monochromatic). For S1, the monochromatic limit correspond to a $\pi$-phase ring. This phase ring solution is easily understood in the monochromatic case, with the simple reasoning at the center of the pupil, a in Sect. 2. For S2, the monochromatic limit solution is the PARRC solution. The defocus tends towards zero, and the amplitude apodization correspond to the analytical prolate solution. The mask size is also the theoretically predicted value for PARRC (Soummer et al. 2003). The existence of two solutions S1 and $\mathrm{S} 2$ can then be understood as the polychromatic generalization of the two monochromatic solutions: a $\pi$ phase mask or a $\pi$ phase ring. This explains also the greater interest for S2, since it is related to the PARRC which permits the total extinction in the monochromatic case.

\subsection{Effect of central obscuration}

The R\&R Phase Mask coronagraph does not depend much on pupil shape (Guyon \& Roddier 2002) and can even be used with arbitrary filled or segmented apertures. This is because the wave diffracted by the mask (i.e. the subtractive coronagraphic wavefront) is a smooth curve that does not depend on the aperture shape. The DZPM is based on the same principle as the $R \& R$ and benefits from the same effect. This is a great advantage over some of the other coronagraphic techniques, for example considering the hexagonally segmented NGST pupil.

We present here a simulation for $14 \%$ obscuration (similar to the ESO-VLT pupil). The central obscuration is simply modelled by the secondary mirror, ignoring the effect of secondary spiders. 
Table 2. Results and performances of the optimization with a central obscuration of $14 \%$. The results are similar to those obtain without obscuration.

\begin{tabular}{llllllllllll}
\hline \hline Technique & $\begin{array}{l}\text { Band } \\
(\%)\end{array}$ & $\begin{array}{l}\text { Apod. } \\
T\end{array}$ & $\begin{array}{l}\text { Mask 1 } \\
a_{1}\end{array}$ & $\begin{array}{l}\text { Mask 2 } \\
a_{2}\end{array}$ & $\begin{array}{l}\text { OPD1 } \\
2 z_{1}\end{array}$ & $\begin{array}{l}\text { OPD2 } \\
2 z_{2}\end{array}$ & $\begin{array}{l}\text { Defoc. } \\
\text { Res. Ener. }\end{array}$ & $\begin{array}{l}\text { level @ } \\
1.5 \lambda / D\end{array}$ & $\begin{array}{l}\text { level @ } \\
3 \lambda / D\end{array}$ & $\begin{array}{l}\text { level @ } \\
7 \lambda / D\end{array}$ \\
\hline DZPM S2 & $40 \%$ & $58.2 \%$ & $0.91 / \mathrm{D}$ & $1.57 / D$ & $0.34 \lambda_{0}$ & $0.73 \lambda_{0}$ & -0.21 & $1.8 \times 10^{-3}$ & $3.3 \times 10^{-5}$ & $4.4 \times 10^{-6}$ & $3.6 \times 10^{-7}$ \\
DZPM S2 & $20 \%$ & $58.0 \%$ & $0.95 / \mathrm{D}$ & $1.59 / D$ & $0.34 \lambda_{0}$ & $0.74 \lambda_{0}$ & -0.23 & $2.3 \times 10^{-4}$ & $1.2 \times 10^{-5}$ & $1.4 \times 10^{-6}$ & $1.0 \times 10^{-7}$ \\
\hline
\end{tabular}
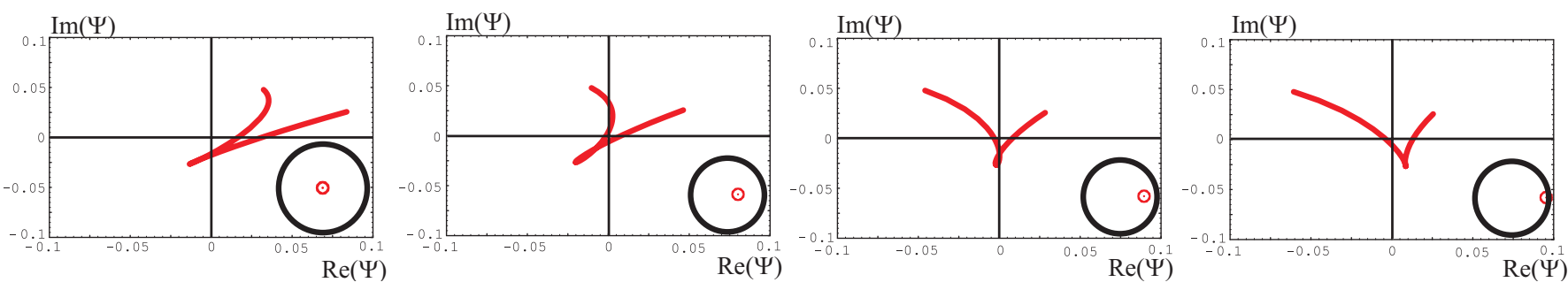

Fig. 15. Illustration of the amplitude in the complex plane, for different positions in the coronagraphic pupil, to be compared with Fig. 8. Here the curves correspond to S2, with same parameters as Fig. 14 (complex apodization and $40 \%$ bandwidth).

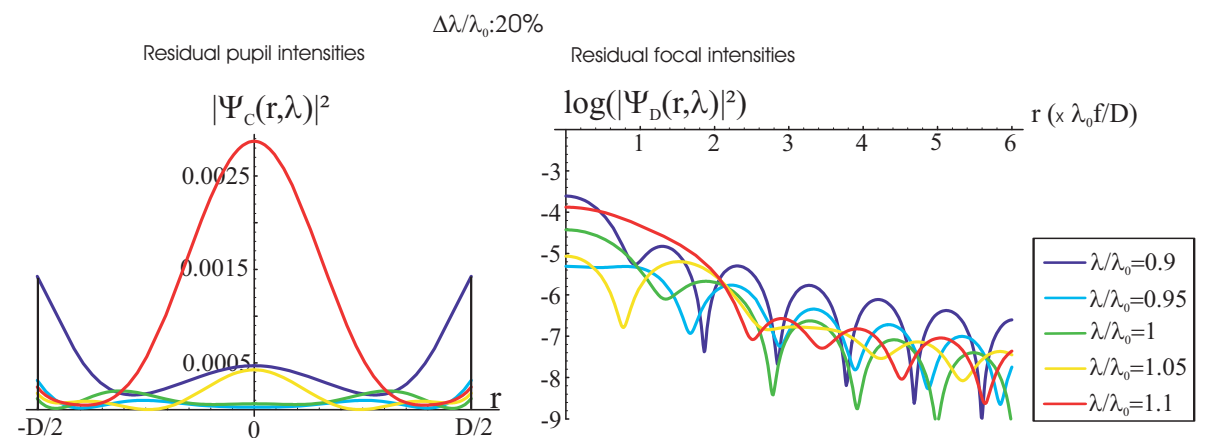

Fig. 16. Results for the solution S2, using apodization and defocus. Left: intensities in the coronagraphic pupil inside the Lyot Stop for several wavelengths within the $20 \%$ band. Right: radial cuts of the residual intensities of a star in log scale (colored in the on-line version). The intensities are normalized at the origin to directly read the coronagraphic extinction ratio at a given angular distance from the on-axis unresolved star.
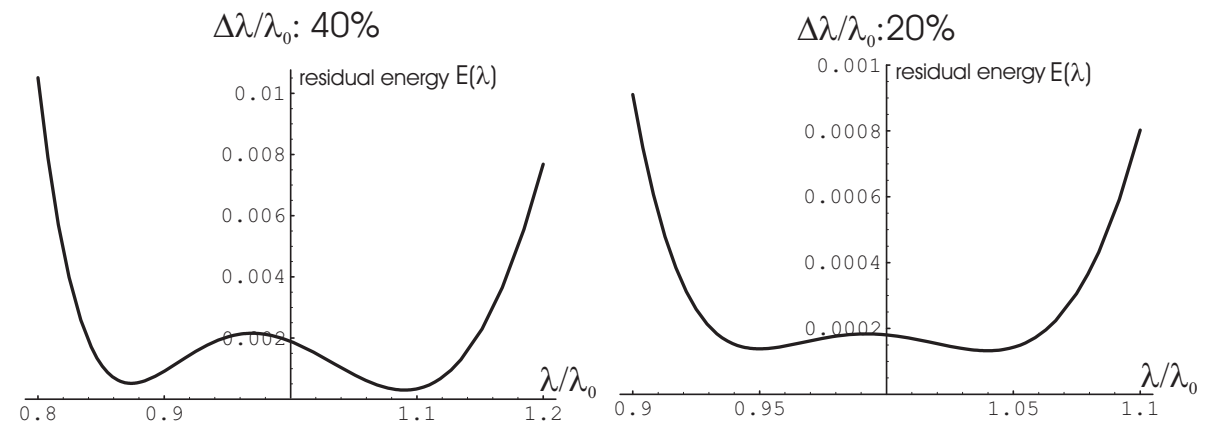

Fig. 17. Results for the S2 using apodization + defocus: residual star energy integrated inside the exit pupil and normalized to the apodizer throughput $T$, as a function of the wavelength. $E(\lambda)=$ $\frac{1}{T} \int_{0}^{D / 2} 2 \pi r\left|\Psi_{\mathrm{C}}(r, \lambda)\right|^{2} \mathrm{~d} r$. This result can be compared to Fig.2 corresponding to the PARRC case.
This procedure preserves the radial symmetry of the pupil (Soummer et al. 2003), allowing an adaptation of the formalism of Sect. 3.1. To extend the method to arbitrary pupil shapes, a fully numerical approach is required, but this is outside the scope of the present paper.

The results and performance calculations in the case of central obscuration are summarized in Table 2: the performance is similar to the case without obscuration. Illustrations are given in Figs. 20 and 21, comparing the intensities before and after the Lyot stop.

\subsection{Triple zone}

With the single point reasoning, three vectors are sufficient to obtain a zero complex amplitude. However, considering Fig. 15 we see that the three vector compensation is not perfect everywhere inside the coronagraphic pupil. Introducing a fourth vector provides further degrees of freedom for the optimization, and may improve the nulling.

This can be done by adding a second annular zone. The formalism can be adapted straightforwardly from the dual zone configuration, introducing a third diameter $d_{3}$ and phase step $z_{3}$. Several solutions exist and the complexity is largely increased for the optimization. We give some results, summarized in Table 3. A gain exists, roughly of a factor 2 to 3 . However the realization (Fig. 22) of a the mask would be more difficult.

This improvement of the technique using three zones instead of two may suggest one to search for a continuous phase shift. It is not excluded that further improvement of the technique may be obtained in this case. 
Polychromatic Residual focal intensities

$\Delta \lambda / \lambda_{0}: 40 \%$

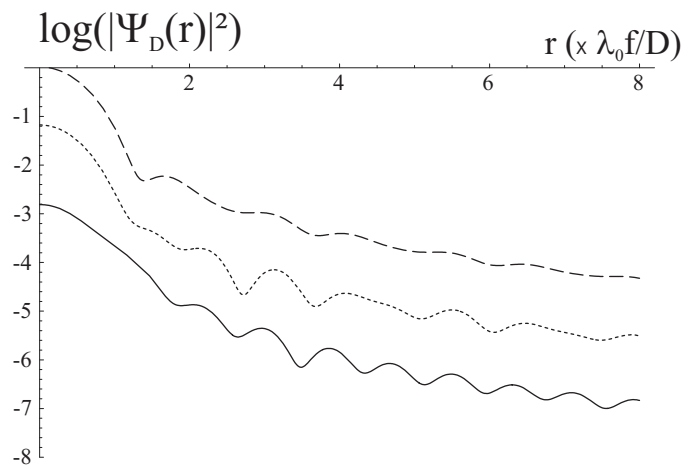


Table 3. Results and performances of the optimization with a central obscuration of $14 \%$. The results are similar to those obtain without obscuration.

\begin{tabular}{lllllllllllll}
\hline \hline Band & Apod. & Mask 1 & Mask 2 & Mask 3 & OPD1 & OPD2 & OPD3 & Defoc. Res. Ener. level @ & level @ & level @ \\
$(\%)$ & $T$ & $a_{1}$ & $a_{2}$ & $a_{3}$ & $2 z_{1}$ & $2 z_{2}$ & $2 z_{2}$ & $\chi$ & $R$ & $1.5 \lambda / D$ & $3 \lambda / D$ & $7 \lambda / D$ \\
\hline $40 \%$ & $48.1 \%$ & $0.70 / D$ & $1.26 / D$ & $1.70 / D$ & $0.22 \lambda_{0}$ & $0.54 \lambda_{0}$ & $0.84 \lambda_{0}$ & -0.20 & $8.6 \times 10^{-4}$ & $1.8 \times 10^{-5}$ & $1.65 \times 10^{-6}$ & $1.0 \times 10^{-7}$ \\
$20 \%$ & $48.6 \%$ & $0.65 / D$ & $1.22 / D$ & $1.69 / D$ & $0.20 \lambda_{0}$ & $0.51 \lambda_{0}$ & $0.82 \lambda_{0}$ & -0.22 & $1.2 \times 10^{-4}$ & $4.4 \times 10^{-6}$ & $5.6 \times 10^{-7}$ & $5 \times 10^{-8}$ \\
\hline
\end{tabular}

Polychromatic residual focal intensities (with central obscuration )

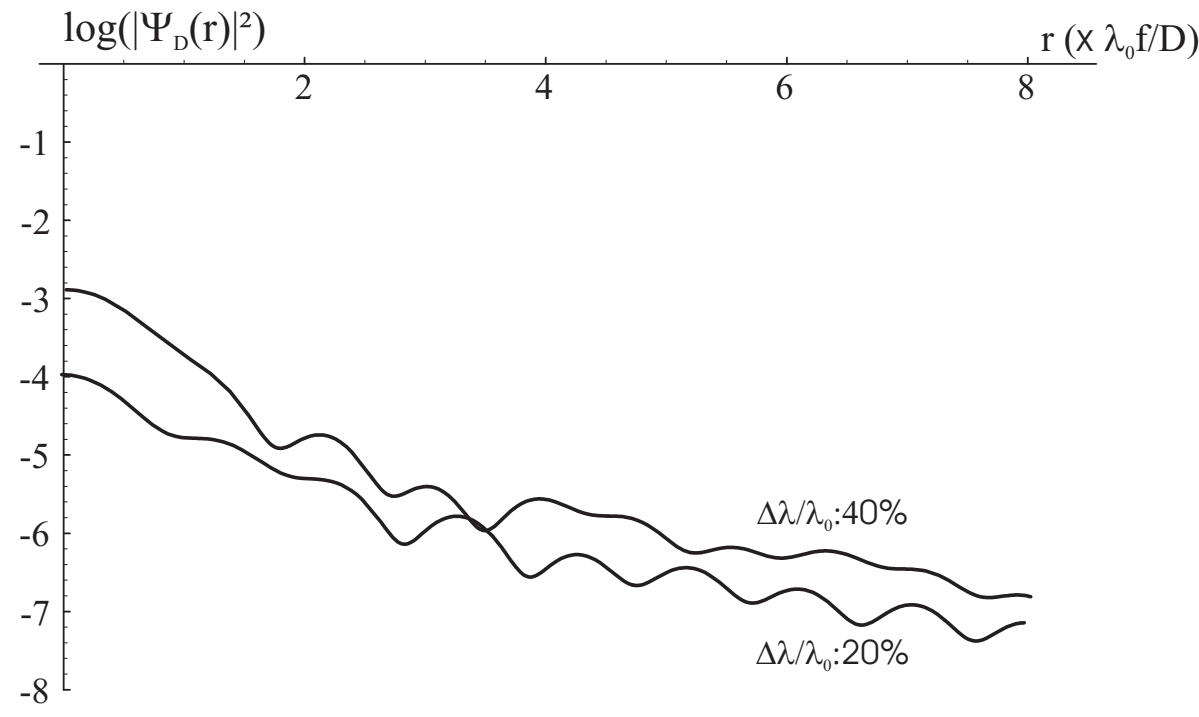

Fig. 21. Illustration of the polychromatic residual focal intensities for solution S2 (bandwidth 20\%), with complex apodization (apodization + defocus) and a cental obscuration of $14 \%$. The intensities are integrated over the bandwidth. The performance is similar to that of the full aperture case.

\section{Conclusion}

In this paper, we have proposed a new coronagraphic concept that is an evolution of the apodized Roddier \& Roddier Phase Mask technique. With this approach we include the chromatism problems in the analysis of the Phase Mask principle. Considerations of achromatic nulling at the center of the coronagraphic pupil lead to the introduction of a second, doughnutshaped phase mask zone, surrounding the original phase disk. In this case of a dual-zone phase mask, the required phase shifts are no longer $\pi$. The overall mask diameter is a little bit larger than the R\&R Phase Mask, of the order of $60 \%$ of the diameter of the Airy core.

As expected, entrance pupil apodization is also needed for the DZPM to obtain a satisfactory nulling. The analytical prolate spheroidal apodizations cannot be applied to the DZPM and we have performed a numerical study instead. However, the amplitude apodization can be well described by a simple polynomial expansion, that can be limited to two terms (fourth order). The interest for complex apodization has been investigated, and it appeared that a slight defocus on the entrance pupil (i.e. the DZPM is not exactly in the focal plane) permits a considerable improvement of the technique.

The optimal solutions have been obtained numerically, using classical minimization techniques. All the computations in this paper have been made semi-analytically, taking advantage of the formalism developed for coronagraphy with circular apertures (Soummer et al. 2003). For arbitrary pupil shapes

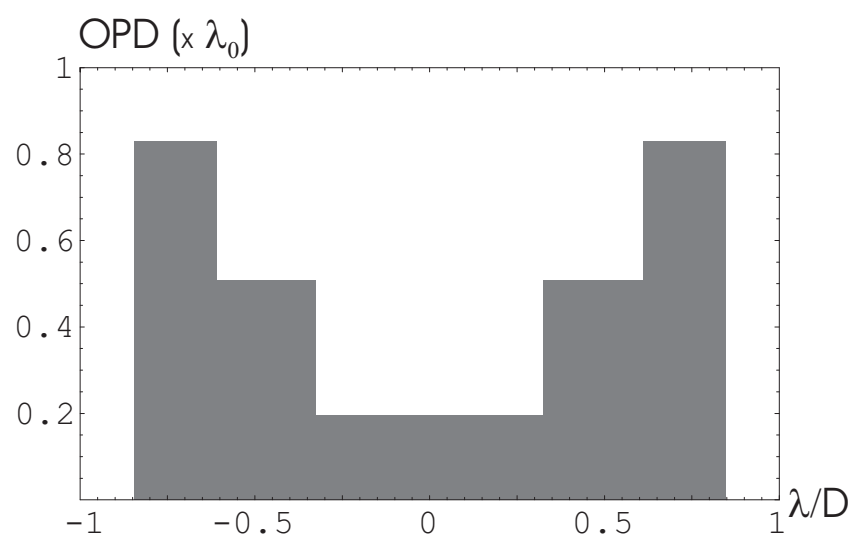

Fig. 22. Representation of the Optical path difference in units of the central wavelength $\lambda_{0}$ for the triple zone solution for $40 \%$.

including segment edges and secondary mirror spiders, a fully numerical approach must be developed. This is trivial although computationally heavier.

Although total polychromatic extinction has not been achieved, a very high coronagraphic extinction ratio can be reached. One of the main advantages of the technique is to accept centrally obscured apertures without performance degradation. For example, with a 14\% obscuration (VLTlike) and $40 \%$ bandwidth, the mean polychromatic intensity 
dynamic is of $2.5 \times 10^{4}$ at $1.5 \lambda / D, 2.5 \times 10^{5}$ at $3 \lambda / D$ and $2.7 \times 10^{6}$ at $7 \lambda / D$.

For the NGST/MIRI coronagraph with a hexagonally segmented telescope pupil (TRW concept), a similar performance can be expected if apodization could be applied. If not, a more limited performance can be expected (see Table 1, DZPM without apodization).

The technique is well suited for very large bandwidths: the coronagraphic performance remains high even for a band of $40 \%$. This point makes the technique particularly well adapted to scientific objectives for which the chromatic characterization is essential (detection and characterization of Jupiterlike planets, debris disks etc.). The technique can be used for smaller bandwidths, and will always give better performance than the R\&R reference without chromatic compensation. It is also interesting to consider the possibility of optimizing two narrow bands separately, taking advantage of the two minima observed in the residual energy curve. In particular, this would be very interesting for differential imaging. We have not illustrated this aspect here since the main characteristic is its interest for large bandwidths.

Additional gain can be brought to the technique using an optimized Lyot Stop (with a possible improvement by a factor 1.5 to 2 , from our tests).

Another advantage of the proposed technique is its relative simplicity of practical realization. In this paper, we have considered the case of a reflective phase mask, but the same principle can be applied to transmissive masks, by including the dispersion laws in the analytical relations. Using different materials for each zone or even combinations of materials adds degrees of freedom and may improve the performance further.

Introducing a third zone improves the achromatization and the gain reaches roughly a factor 3 for a $40 \%$ band. However, the complexity is much higher from the point of view of its realization. This suggests that the use of a continuous phase shifting mask may improve the performance further. This will be the subject of future studies.

Several points remain to be studied, in particular the sensitivity to deviations from the perfect case, and a comparison with the performance of other coronagraphic techniques. The analysis of the performance, applied to realistic scientific objects, remains the most essential point to be studied.
Acknowledgements. The authors are thankful to Olivier Guyon for interesting discussions, and to the referee for his very careful reading of the manuscript and many useful comments.

\section{References}

Abe, L., Domiciano de Souza, J., Vakili, F., \& Gay, J. 2003, A\&A, in press

Abe, L., Vakili, F., \& Boccaletti, A. 2001, A\&A, 374, 1161

Aime, C., \& Soummer, R. 2002, in Astronomy With High Contrast Imaging: from planetary systems to avtive galactic nuclei, ed. C. Aime, \& R. Soummer (EAS Publication Series)

Aime, C., Soummer, R., \& Ferrari, A. 2001, A\&A, 379, 697

Aime, C., Soummer, R., \& Ferrari, A. 2002, A\&A, 389, 334

Baudoz, P. 1999, Ph.D. Thesis, University of Nice Sophia-Antipolis, France

Baudoz, P., Rabbia, Y., \& Gay, J. 2000a, A\&AS, 141, 319

Baudoz, P., Rabbia, Y., Gay, J., et al. 2000b, A\&AS, 145, 341

Gay, J., \& Rabbia, Y. 1996, C. R. Acad. Sci Paris, 322 Série II b, 265

Goodman, J. 1996, Introduction to Fourier Optics, ed. M. G. Hill

Guyon, O., Roddier, C., Graves, J. E., et al. 1999, PASP, 111, 1321

Guyon, O., \& Roddier, F. 2000, in Proc. of SPIE, 4006, 377

Guyon, O., \& Roddier, F. 2002, A\&A, 391, 379

Llyod, J., Gavel, D., Graham, J., Sivaramakrishnan, A., \& Voit, G. 2002, in High Contrast Imaging for Exoplanet Detection, ed. A. B. Schultz, \& R. G. Lyon, Proc. SPIE, 4860

Mawet, D., Lenaerts, C., Moreau, V., et al. 2002, in Astronomy With High Contrast Imaging: from planetary systems to avtive galactic nuclei, ed. C. Aime, \& R. Soummer (EAS Publication Ser.)

Nisenson, P., \& Papaliolios, C. 2001, ApJ, 549

Riaud, P., Boccaletti, A., Rouan, D., Lemarquis, F., \& Labeyrie, A. 2001, PASP, 113, 1145

Roddier, C., Roddier, F., Martin, F., Baranne, A., \& Brun, R. 1980, J. of Opt., 11, 149

Roddier, F., \& Roddier, C. 1997, PASP, 109, 815

Rouan, D., Riaud, P., Boccaletti, A., Clénet, Y., \& Labeyrie, A. 2000, PASP, 112, 1479

Soummer, R., Aime, C., \& Falloon, P. 2002, in Astronomy With High Contrast Imaging: from planetary systems to avtive galactic nuclei, ed. C. Aime, \& R. Soummer (EAS Publication Ser.)

Soummer, R., Aime, C., \& Falloon, P. 2003, A\&A, 397, 1161

Wynne, C. G. 1979, Opt. Commun., 28, 21 